\title{
Performance analysis of wavelength multiplexed SAC OCDMA codes in beat noise mitigation in SAC OCDMA systems
}

\author{
A. M. Alhassan \\ ahmed7060@yahoo.com
}

\section{N. Badruddin}

\section{N. M. Saad}

\section{S. A. Aljunid}

\begin{abstract}
Department of Electrical and Electronic Engineering, Unversiti Teknologi Petronas, Bandar Seri Iskandar, 31750 Tronoh, Perak, Malaysia

Department of Electrical and Electronic Engineering, Unversiti Teknologi Petronas, Bandar Seri Iskandar, 31750 Tronoh, Perak, Malaysia

Department of Electrical and Electronic Engineering, Unversiti Teknologi Petronas, Bandar Seri Iskandar, 31750 Tronoh, Perak, Malaysia

School of Computer and Communication Engineering, Universiti Malaysia Perlis, Tingkat 1, Kampus Tetap Pauh Putra 02600 Arau, Perlis, Malaysia
\end{abstract}

In this paper we investigate the use of wavelength multiplexed spectral amplitude coding (WM SAC) codes in beat noise mitigation in coherent source SAC OCDMA systems. A WM SAC code is a low weight SAC code, where the whole code structure is repeated diagonally (once or more) in the wavelength domain to achieve the same cardinality as a higher weight SAC code. Results show that for highly populated networks, the WM SAC codes provide better performance than SAC codes. However, for small number of active users the situation is reversed. Apart from their promising improvement in performance, these codes are more flexible and impose less complexity on the system design than their SAC counterparts.

[DOI: http://dx.doi.org/10.2971/jeos.2013.13040]

Keywords: Optical code division multiple access, spectral amplitude coding, wavelength multiplexed, coherent sources

\section{INTRODUCTION}

The development of high speed access networks is gaining extreme importance to provide data-hungry user applications with the required data rates. Optical fibers are now deployed in the last mile to achieve that objective. Most of today's access network technologies adopt time division multiple access (TDMA) as the main multi access scheme to serve multiple number of simultaneous users. However, to achieve higher data rates for future applications, researchers are taking into consideration both wavelength division multiple access (WDMA) and optical code division multiple access (OCDMA) schemes.

OCDMA is a multi access scheme that is based on assigning a unique code signature to each user. By sending/receiving these unique signatures, simultaneous access between users can be achieved. The major impairment of OCDMA systems is that they suffer from multi access interference (MAI) which increases with the increase in the number of active users [1]. Different OCDMA techniques mitigate the MAI using different approaches.

A simple and low cost scheme to cancel the MAI is to adopt spectral amplitude coding (SAC) to OCDMA [2,3]. Additional to its MAI cancelation feature, SAC OCDMA deploys low cost broadband incoherent sources such as light emitting diodes (LED) and amplified spontaneous emission (ASE). The broadband nature of these sources makes them a natural choice for SAC OCDMA operation [4]-[14]. However, the major im- pairment of such sources is intensity noise (IN) or phase induced intensity noise (PIIN) [15]. Intensity noise is the result of the square law photodetection of incoherent sources. A major characteristic of this noise is its signal dependency, which increases with the signal strength creating an upper limit in the performance.

To overcome the performance limitations endured by incoherent sources and increase the capacity of SAC OCDMA systems, researchers investigated the use of coherent sources as an alternative to the incoherent counterparts [16]-[20]. Ayotte and Rusch performed an intensive study of the use of coherent sources to increase the capacity of SAC OCDMA [17]. They showed that coherent sources suffer from beat noise, which is the result of laser signals beating together. They investigated three different laser source configurations and found that the use a well controlled multi laser source will outperform any other source configuration, for a highly populated network.

In [21], Yang proposed a hybrid wavelength division multiplexing/spectral amplitude coding (WDM/SAC) to reduce the PIIN in incoherent SAC OCDMA system, where he used wavelength multiplexed spectral amplitude coding (WM SAC) codes. Apart from its PIIN mitigation abilities, the WM SAC is lower in cost and complexity than the conventional SAC OCDMA [21]. In this paper we investigate the use of WM SAC codes in beat noise mitigation when coherent sources are deployed. We will show that by applying WM 


\begin{tabular}{|c|c|c|c|c|c|c|c|c|c|c|c|c|c|c|}
\hline No. & \multicolumn{10}{|c|}{ Codewords } \\
\hline $\mathbf{1}$ & 0 & 0 & 0 & 0 & 0 & 0 & 0 & 0 & 0 & 0 & 1 & 0 & 1 & 1 \\
\hline $\mathbf{2}$ & 0 & 0 & 0 & 0 & 0 & 0 & 0 & 0 & 0 & 1 & 0 & 1 & 1 & 0 \\
\hline $\mathbf{3}$ & 0 & 0 & 0 & 0 & 0 & 0 & 0 & 0 & 1 & 0 & 1 & 1 & 0 & 0 \\
\hline $\mathbf{4}$ & 0 & 0 & 0 & 0 & 0 & 0 & 0 & 1 & 0 & 1 & 1 & 0 & 0 & 0 \\
\hline $\mathbf{5}$ & 0 & 0 & 0 & 0 & 0 & 0 & 0 & 0 & 1 & 1 & 0 & 0 & 0 & 1 \\
\hline $\mathbf{6}$ & 0 & 0 & 0 & 0 & 0 & 0 & 0 & 1 & 1 & 0 & 0 & 0 & 1 & 0 \\
\hline $\mathbf{7}$ & 0 & 0 & 0 & 0 & 0 & 0 & 0 & 1 & 0 & 0 & 0 & 1 & 0 & 1 \\
\hline $\mathbf{8}$ & 0 & 0 & 0 & 1 & 0 & 1 & 1 & 0 & 0 & 0 & 0 & 0 & 0 & 0 \\
\hline $\mathbf{9}$ & 0 & 0 & 1 & 0 & 1 & 1 & 0 & 0 & 0 & 0 & 0 & 0 & 0 & 0 \\
\hline $\mathbf{1 0}$ & 0 & 1 & 0 & 1 & 1 & 0 & 0 & 0 & 0 & 0 & 0 & 0 & 0 & 0 \\
\hline $\mathbf{1 1}$ & 1 & 0 & 1 & 1 & 0 & 0 & 0 & 0 & 0 & 0 & 0 & 0 & 0 & 0 \\
\hline $\mathbf{1 2}$ & 0 & 1 & 1 & 0 & 0 & 0 & 1 & 0 & 0 & 0 & 0 & 0 & 0 & 0 \\
\hline $\mathbf{1 3}$ & 1 & 1 & 0 & 0 & 0 & 1 & 0 & 0 & 0 & 0 & 0 & 0 & 0 & 0 \\
\hline $\mathbf{1 4}$ & 1 & 0 & 0 & 0 & 1 & 0 & 1 & 0 & 0 & 0 & 0 & 0 & 0 & 0 \\
\hline
\end{tabular}

TABLE 1 A WM SAC BIBD code [21].

SAC, the performance will improve for a highly loaded network, whereas for a lightly load network, SAC codes give better results. The rest of the paper is organized as follows; in Section 2 we review the WM SAC structure. In Section 3 we perform a performance analysis for the WM SAC for coherent sources. A simulation setup is given in Section 4 for both the WM SAC and SAC schemes. In Section 5 the results are discussed, and finally the paper is concluded in Section 6.

\section{WAVELENGTH MULTIPLEXED SPECTRAL AMPLITUDE CODING (WM SAC) OCDMA SYSTEM}

Table 1 show a simple WM SAC code with two groups $(S=2)$ [21]. The basic building block of the code is a balanced incomplete block design (BIBD) code with prime power of two (weight of three). The WM SAC code is generated by repeating the basic building block diagonally in wavelength two or more times depending on the number of groups. The in phase cross correlation between codes within the same group is one $(\lambda=1)$, and between codes from different groups is zero $(\lambda=0)$.

The structure of the transmitter for the WM SAC system shown in Figure 1(a) is similar to the conventional SAC OCDMA transmitter [5]. However, the receiver structure has some minor modifications. The receivers for WM SAC are designed to only detect (and cancel) codes that are within the same group. In Figure 1(b) the decoder (DEC) branch only detects the intended signal spectrum and overlapping spectra from other interferers (within the same group). The complementary decoder (CDEC) branch detects the complementary spectrum of the intended user within the spectra of that group. Interferers that are from different groups are not detected by the DEC filters or CDEC filters. One major advantage of this receiver's design is its filter design is less complex than the SAC receiver design for the same number of users. We note the structures for both decoder and complementary decoder given in Figure 1(b) use fiber Bragg grating $(\mathrm{FBG})$ in transmission. Different filter technologies can be used, but some changes must be taken into consideration, e.g. as in [21].

\section{PERFORMANCE ANALYSIS OF THE WM SAC}

In this section we evaluate the performance of the WM SAC scheme in beat noise reduction for coherent sources. In our evaluation we take three different coherent sources into consideration- A centralized shared multi laser source, a uniformly distributed multi laser source and a precisely controlled multi laser source given in Figure 2 [17]. To predict the performance of our system, we begin by finding the characteristic functions and the probability density functions of the intensity for all three different source configurations.

\subsection{Characteristic functions for different source configurations:}

\subsubsection{Shared multi laser source:}

In this case a centralized shared multi laser source is deployed. For each spectral bin all the laser central frequencies will be the same. However, due to the propagation distances, the phase and polarization of each laser will differ depending on the user's position from the source. We assume that the phase and polarization angles are random variables that have uniform distribution. Figure 2(a) shows the laser occupation in this case.

In order to compute bit error rate (BER) of our system, we need to derive expressions for both the probability density function (pdf) and its Fourier transform the characteristics function. Since there is no analytical expression for the intensity of two or more lasers that have random phase and polarization [17], we use Monte Carlo simulation to obtain the characteristic function and the pdf [17]. 


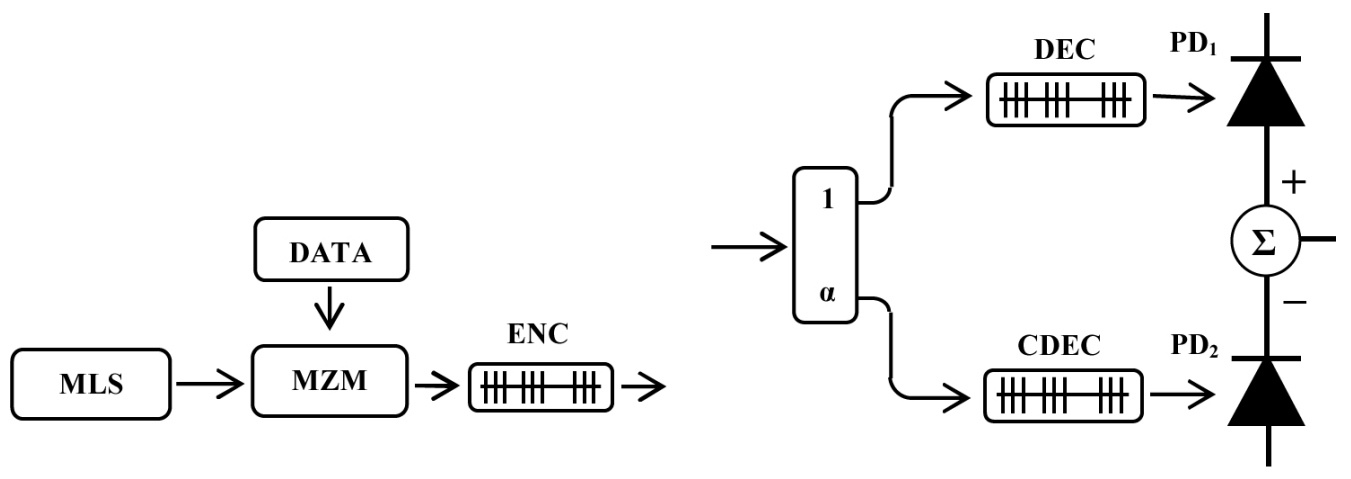

(a)

(b)

FIG. 1 The structure of the WM SAC (a) Transmitter (b) Receiver, MLS: multi laser source, MZN: Mach-Zehnder modulator, ENC: encoder, DEC: decoder, CDEC: complementary decoder, PD: photodiode.

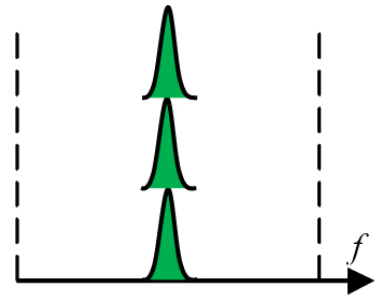

(a)

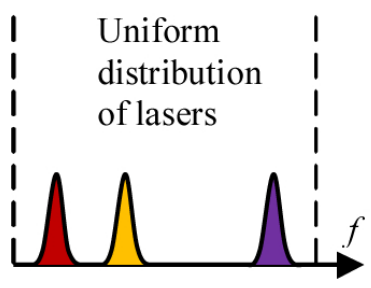

(b)

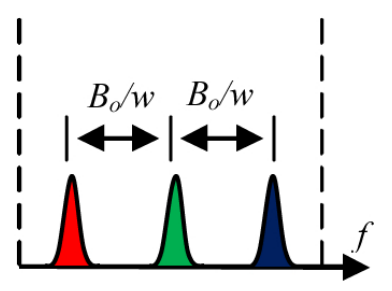

(c)
FIG. 2 Spectral bin occupation for the three coherent source configurations. (a) Centralized shared multi laser source. (b) Uniformly distributed multi laser source. (c) Controlled central frequency multi laser source [17].

To compute the characteristic function we follow the same procedure as in [17]. An overview of the method is explained below.

The electrical field for a single realization $i$ of phase and polarization for user $u$ can be expressed as:

$$
E_{u, i}=\left[\begin{array}{c}
\sin \left(\beta_{u, i}\right) \exp \left(\delta_{u, i}\right) \\
\cos \left(\beta_{u, i}\right) \exp \left(\phi_{u, i}\right)
\end{array}\right] \exp \left(j \omega_{u} t\right)
$$

where $\beta_{u, i}$ is the angle of polarization, $\delta_{u, i}$ and $\phi_{u, i}$ are the phases of the two polarization states, and $\omega_{u}$ is the angular frequency. All $\beta, \delta$, and $\phi$ are uniformly distributed random variables over the period $[0,2 \pi]$.

The generated intensity for $L$ different users for a single realization of phase and polarization can be given by:

$$
I_{L, i}=\left(\sum_{u=1}^{L} E_{u, i}\right)\left(\sum_{u=1}^{L} E_{u, i}^{*}\right)
$$

Assuming that all the different realizations have the same probability, we compute the empirical distribution for $10^{7}$ dif- ferent realizations for the phase and polarization angles. The cumulative distribution function (cdf) can be expressed as:

$$
F(x \mid L)=\frac{1}{10^{7}} \sum_{i=1}^{10^{7}} 1_{\left\{I_{L, i} \leq x\right\}}
$$

where $1_{\left\{I_{L, i} \leq x\right\}}$ equal one if the inequality is true, and zero if the inequality is false.

From the cdf, we get the pdf through differentiation:

$$
f(x \mid L)=\frac{d(F(x \mid L))}{d x}
$$

and through Fourier transform we get the characteristics function:

$$
\Omega_{I}(\omega \mid L)=\int_{-\infty}^{\infty} f(x \mid L) e^{j \omega x} d x
$$

For more than 5 lasers a Gamma approximation for the pdf can be used [17].

\subsubsection{Uniformly distributed multi laser source:}

In this case each user is equipped with its own multi laser source. The assumption is that for each spectral bin, the lasers' central frequencies will fall within that bin's bandwidth $\left(B_{0}\right)$. However, due to manufacturing the laser central frequencies will be uniformly distributed within the bin. Figure 2(b) shows the distribution of lasers within a spectral bin. Beat noise can only occur when two or more lasers are spaced from each other at no greater distance than the electrical bandwidth $\left(B_{e}\right)$. This can be mathematically interpreted by dividing the bin spectral width $\left(B_{0}\right)$ into $r$ subdivisions, where each subdivision equals to the electrical bandwidth. Beat noise is generated when lasers fall within a specific subdivision.

The characteristics function for each bin in this case is given by [17]:

$$
\Omega_{I}(\omega \mid L)=\sum_{\{m\}} \frac{\left|D_{m}\right|}{r^{L}} \Omega_{I}(\omega \mid L, m)
$$

where $m$ is weight vector and $D_{m}$ is the occupancy vector.

\subsubsection{Controlled multi laser source:}

To further reduce the effect of beat noise, it is assumed that the lasers' central frequencies can be controlled up to a specific 
precision. For any SAC OCDMA code each spectral bin has a maximum of $w$ lasers that fall in that bin. The spectral width $B_{o}$ is divided into $w$ subdivisions and each laser is assigned a different subdivision. The central frequency of each laser is assumed to have a Gaussian distribution, with the mean of the Gaussian curve being the center of the subdivision and the standard deviation being the degree of precision of the laser's manufacturing. A visualization of a bin spectrum is given in Figure 2(c). In this case, the assumption is that beat noise can only occur between two adjacent lasers.

The characteristics function is [17]:

$$
\Omega_{I}(\omega \mid L)=\sum_{B=0}^{L / 2} P(B \mid L) \cdot \Omega_{I}(\omega \mid 2)^{B} \cdot \Omega_{I}(\omega \mid 1)^{L-2 B}
$$

where $B$ is the number of laser pairs beating together, and $P(B \mid L)$ is the probability the $B$ pairs out of $L$ lasers are beating.

\subsection{Bit Error Rate Computation}

We assume that there is $L$ total number of users, the $L$ users are divided into $S$ sets of code sequences, where each set is a BIBD (or any other SAC code) code family. We assume that our desired user is from set number $k$, and there is a total of $l$ interferers sending data " 1 ". Out of the $l$ interferers there are only $l_{k}$ interferers that are actually from set $k$, where:

$$
\sum_{k=1}^{S} l_{k}=l
$$

For a particular pattern of $l$ interferers, we compute the bin occupation numbers, $d_{x}$ and $c d_{y}$ for bin number $x$ in the decoder and bin number $y$ in the complementary decoder, respectively. Using the computed bin occupation numbers we calculate the characteristic function for each spectral bin. The combined intensity of all the spectral bins present in the decoder branch forms the output of the upper photodetector. Meanwhile the combined intensity of the spectral bins present in the complementary decoder branch forms the output of the lower photodetector. To retrieve the intended signal and cancel the MAI, the output of the lower photodetector is subtracted from the output of the upper photodetector. This will determine the final decision statistics of the receiver. In order to find the probability density function of the output, we first compute its characteristic function. The characteristic function is found by multiplying the characteristic functions of the bins in the decoder by the characteristic functions of the bins in the complementary decoder. This is possible due to independent (non-overlapping) nature of the spectral bins.

We would like to note the BER computation in this section is similar to the one given in [17], this is because the same balanced detector is used in both cases. However, the number of interferers $l_{k}$ is different.

The characteristic function of the receiver when the desired user is sending data " 0 " and data " 1 " respectively:

$$
\begin{aligned}
\Omega_{0}\left(\omega \mid U, T, l_{k}\right) & =\left[\Omega_{I}\left(\omega \mid d_{1}\right) \ldots \Omega_{I}\left(\omega \mid d_{x}\right)\right] \\
& \times\left[\Omega_{I}\left(-\omega / \alpha \mid c d_{1}\right) \ldots \Omega_{I}\left(-\omega / \alpha \mid c d_{y}\right)\right]
\end{aligned}
$$

and

$$
\begin{aligned}
\Omega_{1}\left(\omega \mid U, T, l_{k}\right) & =\left[\Omega_{I}\left(\omega \mid d_{1}+1\right) \ldots \Omega_{I}\left(\omega \mid d_{x}+1\right)\right] \\
& \times\left[\Omega_{I}\left(-\omega / \alpha \mid c d_{1}\right) \ldots \Omega_{I}\left(-\omega / \alpha \mid c d_{y}\right)\right]
\end{aligned}
$$

where $U$ is the intended user, $\boldsymbol{T}$ is data vector, which elements $i$ is " 0 " or " 1 " based on what user $i$ is sending.

The expression given in Eq. (9) and (10) represent the characteristic functions for a single realization of the vector $\boldsymbol{T}$. The number of occupation of each frequency bin $\left(d_{x}\right.$ and $\left.c d_{y}\right)$ will vary based on the different combinations of the vector $\boldsymbol{T}$. Assuming all combinations of the vector $\boldsymbol{T}$ are all equiprobable, we average over all possible values as follows.

For the decoder branch, the characteristic function for sending data ' 0 ' is:

$$
\Omega_{0}(\omega \mid U, l)=E_{T}\left\{\Omega_{0}\left(\omega \mid U, T, l_{k}\right)\right\} .
$$

A similar equation can be derived for $\Omega_{1}$.

Assuming that the probability that the users are sending data " 0 " and "1" follow a binomial distribution, the characteristic function when the intended user is sending logic state " 0 " becomes:

$$
\begin{aligned}
& \Omega_{0}(\omega \mid U, L-1) \\
= & \sum_{l=0}^{L-1}\left(\frac{1}{2}\right)^{L-1} \frac{(L-1) !}{l !(L-1-l) !} \Omega_{0}(\omega \mid U, l)
\end{aligned}
$$

A similar equation can be derived for $\Omega_{1}$.

By applying Fourier transform we can obtain the pdfs. Using these pdfs the BER is given by:

$$
\begin{aligned}
& \operatorname{BER}(U, L-1) \\
= & \frac{1}{2}\left(\int_{\gamma}^{\infty} f_{0}(x \mid U, L-1) d x+\int_{-\infty}^{\gamma} f_{1}(x \mid U, L-1) d x\right)
\end{aligned}
$$

where $\gamma$ is the threshold value for the intensity to distinguish between logical level "0" and logical level "1", and its calculated numerically.

\section{SIMULATION SETUP}

\subsection{Monte Carlo simulation:}

The data rates for our simulation are $1.25 \mathrm{~Gb} / \mathrm{s}$ and $10 \mathrm{~Gb} / \mathrm{s}$, and the optical bandwidth is $30 \mathrm{~nm}$. For the data rate $1.25 \mathrm{~Gb} / \mathrm{s}$, a SAC OCDMA BIBD code with cardinality 133 (prime power $(q)=11$ ) is chosen, and for the WM SAC two BIBD codes are considered, the first is a code with cardinality $31(q=5)$ and the second a code with cardinality $7(q=2)$. Since the WM SAC codes can only support 31 and 7 users respectively, we multiplex the code signatures four times $(s=4)$ for the first codes and eighteen times $(s=18)$ for the second code in the wavelength domain generating 124 and 126 different code sequences. For the sake of comparison, only 124 code signatures are taken from the total of the 133 possible code sequences for the SAC code. The bin spectral 
width is $0.23 \mathrm{~nm}$ for both the BD and the WM SAC. For the $10 \mathrm{~Gb} / \mathrm{s}$ data rate a SAC OCDMA code with cardinality of 31 $(q=5)$ is chosen, and for the WM SAC code a BIBD code with cardinality of $7(q=2)$ is considered. For the WM SAC code to support 31 users, the code signature is multiplexed in the frequency domain five times $(s=5)$. The bin spectral width in this case is $0.97 \mathrm{~nm}$.

\subsection{Setup for software simulation:}

In our software simulation we consider a seven user network for both SAC OCDMA and the WM SAC systems. The SAC OCDMA code is a BIBD code with weight of three $(q=2)$ and cardinality of seven. The code for the WM SAC is a BIBD code of weight two $(q=1)$ and cardinality of three. To support seven users the code is repeated three times in the wavelength domain. Table 2 and Table 3 show the code sequences for seven users for SAC OCDMA and the WM SAC networks, respectively.

From Table 2 and Table 3 we can see that the SAC code has seven spectral bins, and the WM SAC code has eight spectral bins. The bin bandwidth for SAC code is set to $0.5 \mathrm{~nm}$, which results in a $3.5 \mathrm{~nm}$ total bandwidth. Meanwhile, for the WM SAC code we set the bin bandwidth to $0.4 \mathrm{~nm}$ resulting in $3.2 \mathrm{~nm}$ total bandwidth.

\begin{tabular}{|c|c|c|c|c|c|c|c|}
\hline User \# & \multicolumn{7}{|c|}{ Code Sequence } \\
\hline User \#1 & 0 & 0 & 0 & 1 & 0 & 1 & 1 \\
\hline User \#2 & 0 & 0 & 1 & 0 & 1 & 1 & 0 \\
\hline User \#3 & 0 & 1 & 0 & 1 & 1 & 0 & 0 \\
\hline User \#4 & 1 & 0 & 1 & 1 & 0 & 0 & 0 \\
\hline User \#5 & 0 & 1 & 1 & 0 & 0 & 0 & 1 \\
\hline User \#6 & 1 & 1 & 0 & 0 & 0 & 1 & 0 \\
\hline User \#7 & 1 & 0 & 0 & 0 & 1 & 0 & 1 \\
\hline
\end{tabular}

TABLE 2 SAC BIBD code $q=2$.

\begin{tabular}{|c|c|c|c|c|c|c|c|c|}
\hline User \# & \multicolumn{10}{|c|}{ Code Sequence } \\
\hline User \#1 & 0 & 0 & 0 & 0 & 0 & 0 & 1 & 1 \\
\hline User \#2 & 0 & 0 & 0 & 0 & 0 & 1 & 1 & 0 \\
\hline User \#3 & 0 & 0 & 0 & 0 & 0 & 1 & 0 & 1 \\
\hline User \#4 & 0 & 0 & 0 & 1 & 1 & 0 & 0 & 0 \\
\hline User \#5 & 0 & 0 & 1 & 1 & 0 & 0 & 0 & 0 \\
\hline User \#6 & 0 & 0 & 1 & 0 & 1 & 0 & 0 & 0 \\
\hline User \#7 & 1 & 1 & 0 & 0 & 0 & 0 & 0 & 0 \\
\hline
\end{tabular}

TABLE 2 WM SAC BIBD code $q=1$.

In our setup we implemented a controlled multi laser source configuration, where we can control the central frequency of each laser. The distribution of the lasers within a bin will depend on the number of lasers and the spectral bandwidth of the bin. From Table 2 we can see that for a full SAC code network, each spectral bin will have a maximum of three active lasers. The lasers are distributed by assigning one of the lasers' central frequencies to the central frequency of the bin, and the other two lasers are placed at $0.2 \mathrm{~nm}$ to the right and left of the centralized laser. An illustration of the laser distribution within a bin for the SAC code is given in Figure 3(a). For the WM SAC code, Table 3 shows that a maximum of two lasers

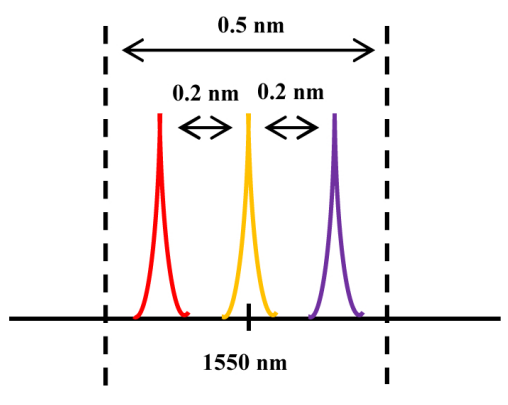

(a)

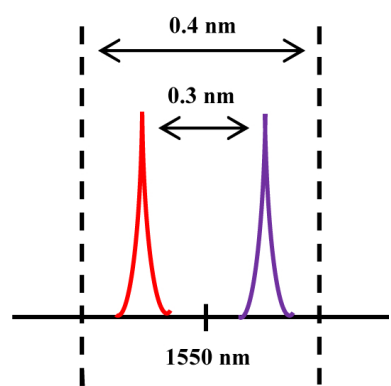

(b)
FIG. 3 Lasers central frequencies distribution within a bin (a) SAC code (b) WM SAC.

can fall within any spectral bin. One of the lasers is placed at $0.15 \mathrm{~nm}$ to the left of the bin central frequency and the other is placed at the same distance to the right of the bin central frequency (the distance between the two lasers is $0.3 \mathrm{~nm}$ ). Figure $3(\mathrm{~b})$ shows the laser distribution for this case. The laser frequency assignment for the whole spectral bandwidth for both the SAC and the WM SAC codes is given in Figure 4 and Figure 5, respectively.

The transmitter for each user consists of three controlled lasers, where each laser's launch power is $0 \mathrm{dBm}$ (unless stated otherwise). The accumulated signal from the three lasers is then modulated with a pseudorandom bit sequence (PRBS) data using a Mach-Zehnder modulator (MZM) with an extinction ratio of $30 \mathrm{dBm}$. We would like to note that since we are using that exact number of lasers in each source there is no need for an encoder in this design.

The receiver's design is similar to the design given in Figure $1(\mathrm{~b})$. The decoder and the complementary decoder are implemented using a fiber bragg gratings (FBG) in reflection with a pass bandwidth of $0.5 \mathrm{~nm}$ for the SAC OCDMA code and $0.35 \mathrm{~nm}$ for the WM SAC code (these values were the optimal values obtained experimentally). The photodiodes in the balanced detector are PIN photodiodes and the receiver's electrical filter is a Bessel filter of the fourth order with an electrical bandwidth equal to $75 \%$ of the data rate.

\section{RESULTS AND DISCUSSION}

\subsection{Monte Carlo simulation}

The BER versus the number of active users for SAC and WM SAC codes at data rate $1.25 \mathrm{~Gb} / \mathrm{s}$ is given in Figure 6 and Figure 7 . Figure 6 shows a SAC code $(q=11, s=1)$ against a WM SAC code $(q=5, s=4)$. Meanwhile, Figure 7 shows the same SAC code against a WM SAC code $(q=2, s=18)$. We can see from both figures that for all three different source configurations, the performance of the WM SAC is worse than the conventional SAC when the number of users is small. This can be explained by noting that a code that has a higher weight will generally perform better than a code with lower weight. However, as the number of users increase, the WM SAC shows better performance than the SAC system. This is mainly due to the better correlation properties of the WM SAC code compared to the SAC code. To highlight the perfor- 


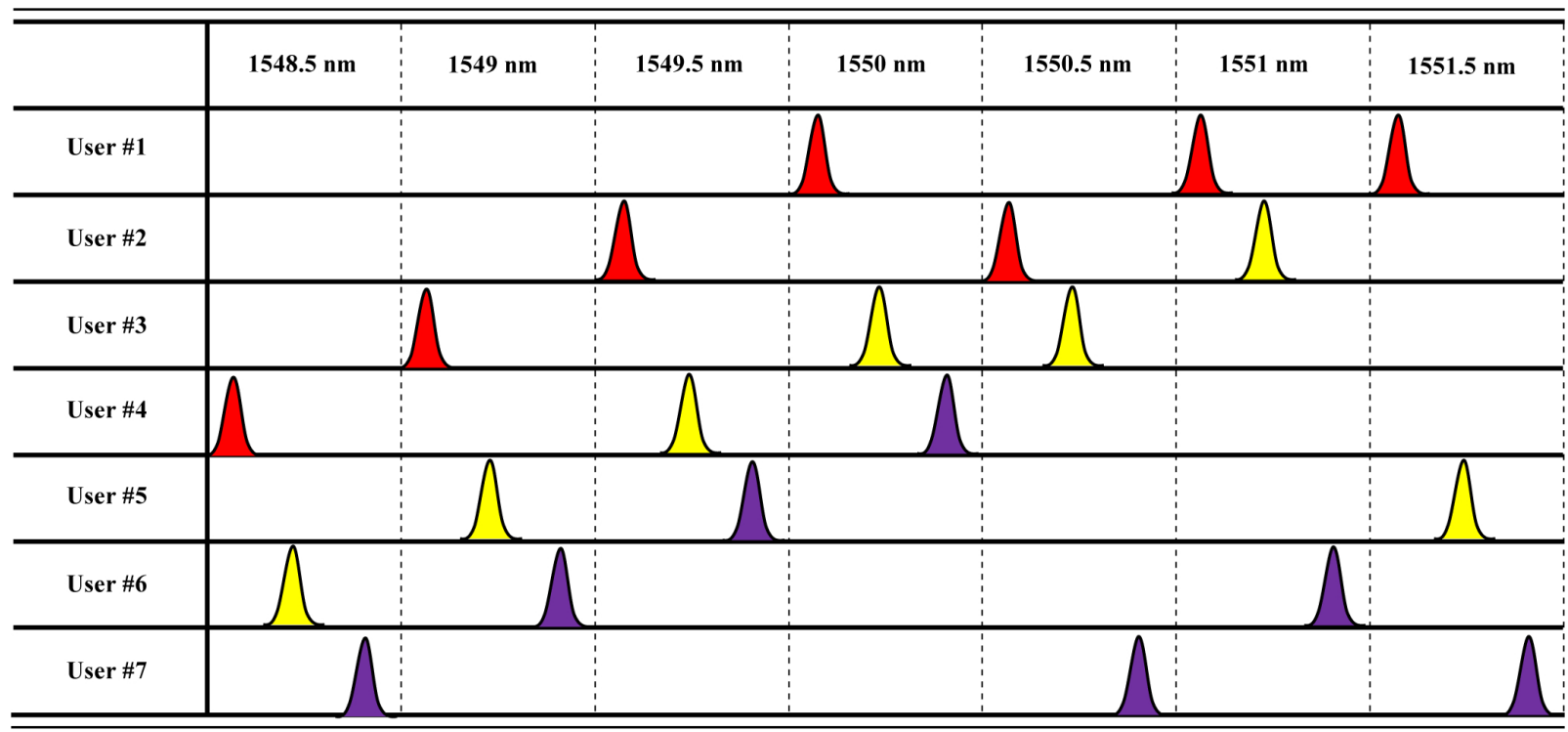

FIG. 4 Total central frequency distributions for BIBD $(q=2)$ SAC code.

\begin{tabular}{|c|c|c|c|c|c|c|c|c|}
\hline & $1548.8 \mathrm{~nm}$ & $1549.2 \mathrm{~nm}$ & $1549.6 \mathrm{~nm}$ & $1550 \mathrm{~nm}$ & $1550.4 \mathrm{~nm}$ & $1550.8 \mathrm{~nm}$ & $1551.2 \mathrm{~nm}$ & $1551.6 \mathrm{~nm}$ \\
\hline \multicolumn{9}{|l|}{ User \#1 } \\
\hline \multicolumn{9}{|l|}{ User \#2 } \\
\hline \multicolumn{9}{|l|}{ User \#3 } \\
\hline \multicolumn{9}{|l|}{ User \#4 } \\
\hline \multicolumn{9}{|l|}{ User \#5 } \\
\hline \multicolumn{9}{|l|}{ User \#6 } \\
\hline User \#7 & & & & & & & & \\
\hline
\end{tabular}

FIG. 5 Total central frequency distributions for $\operatorname{BIBD}(q=1)$ WM SAC code.

mance improvement, for a controlled multi laser source, the SAC code with ultra precision $(0.3 \mathrm{GHz})$ can support 70 active users at a $10^{-12}$ BER. For the same BER, we can see from Figure 6 and Figure 7 that the WM SAC codes $(q=5, s=4)$ and $(q=2, s=18)$ can support up to 80 and 110 active users, respectively, which are a $15 \%$ and $57 \%$ improvement.

Figure 8 shows the BER versus the number active users at $10 \mathrm{~Gb} / \mathrm{s}$ for a SAC code $(q=5, s=1)$ and the WM SAC code $(q=2, s=5)$. We can see from the figure that the number of users is much smaller than the previous simulation, this because of the higher data rate. Also because of the high data rate, only precisely controlled multi laser source configuration is considered. The reason for this is that the shared multi laser source and uniformly distributed multi laser source configurations performance is deeply degraded at such high data rate. By examining the figure we can see that it follows a similar trend as the previous simulation, where when the number of users is small the higher weight SAC code gives a better performance and when the number of users is high the WM
SAC code gives a better performance. As an example, for a BER of $10^{-11}$ the SAC OCDMA code with central frequency precision of $2 \mathrm{GHz}$ can support only 15 users, meanwhile the WM SAC code can support approximately 30 users, two fold of what the SAC OCDMA code can support.

It is interesting to note that the results obtained here for coherent sources agree with the results obtained by Yang [21] when incoherent sources are used. The interpretation for this similarity is that the detected intensity of an incoherent source can be approximated by Gamma pdf, and the detected intensity of overlapping lasers (more than five lasers) can also be approximated by a Gamma pdf.

\subsection{Software simulation}

A comparison between the WM SAC and the SAC OCDMA for seven users at data rate $1.25 \mathrm{~GB} / \mathrm{s}$ is given in Figure 9. From Figure 9 we can observe that for small number of users the conventional system gives better results than the WM 


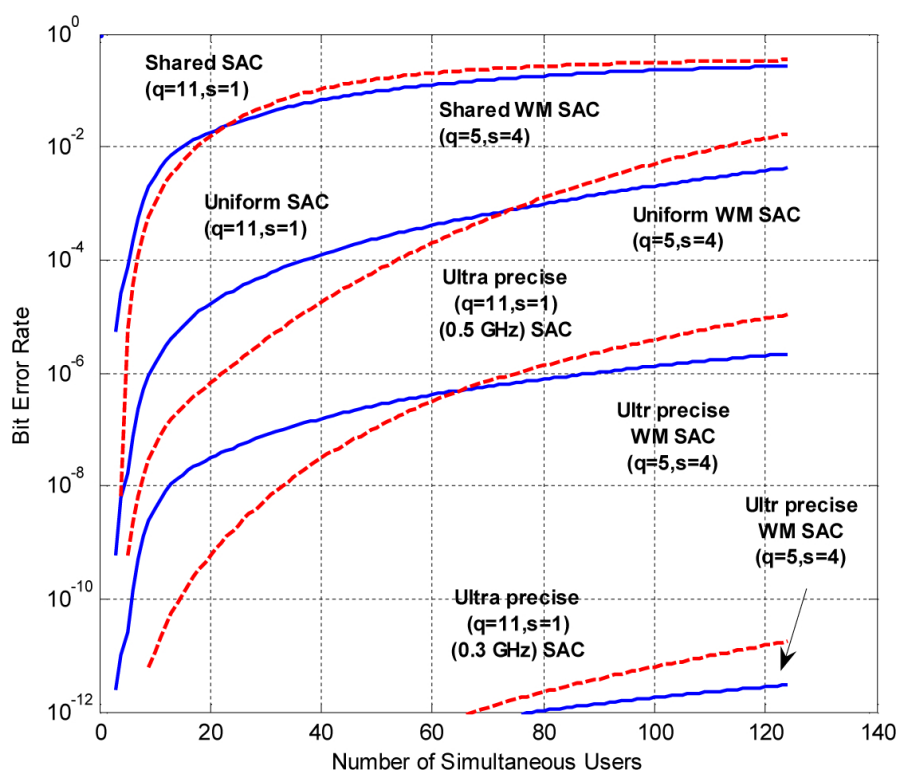

FIG. 6 BER versus the active number of users for SAC code $(q=11, s=1)$ and WM SAC code $(q=5, n=4)$ at data rate of $1.25 \mathrm{~Gb} / \mathrm{s}$.

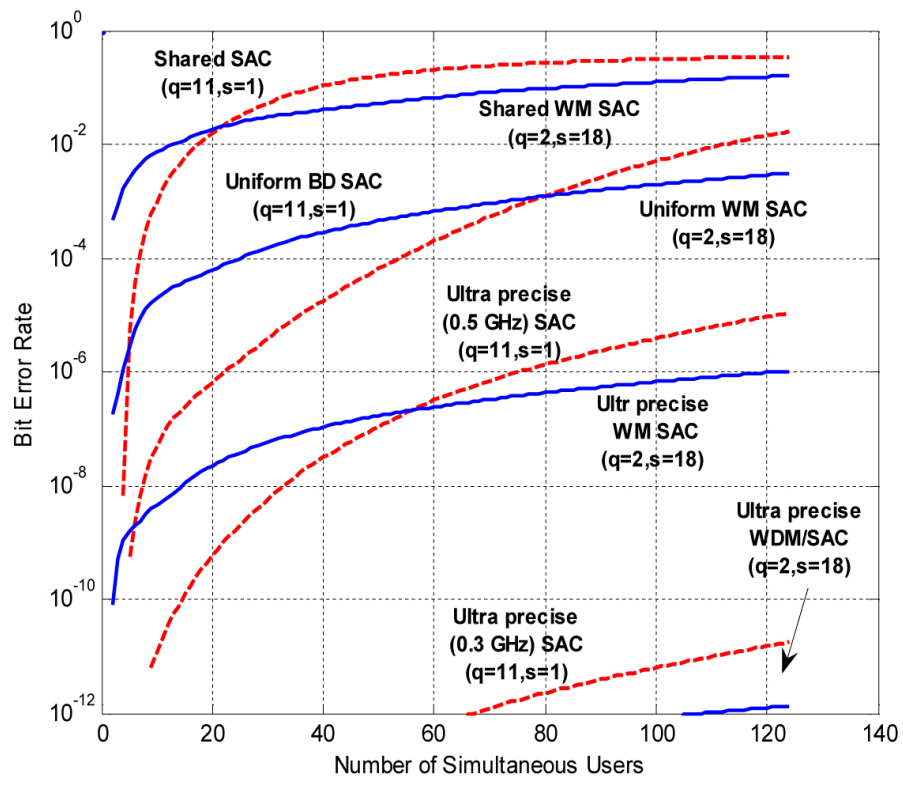

FIG. 7 BER versus the active number of users for SAC code $(q=11, s=1)$ and WM SAC code $(q=2, s=18)$ at data rate of $1.25 \mathrm{~Gb} / \mathrm{s}$.

SAC. However, as the number of users increase (above four users) we find the situation is reversed and the WM SAC outperforms the SAC code. The explanation for this is as follows; for less than four users the beat noise is negligible and thermal noise and shot noise are the main limiting factors. Since the SAC code has a higher weight (stronger signal) than the WM SAC code, the BER is less for the SAC code. However, as the number of users increases, the beat noise becomes the dominant source of noise. Due to its better correlation properties the WM SAC code shows better BER in this case than the SAC code. The eye diagrams for two and six users for both the SAC and the WM SAC codes are given in Figure 9 to compare the quality of the received signal at the two extreme cases. The very low BER (below 10 $0^{-15}$ ) in Figure 9 is due to the small number of users ( 1 to 6 users) where the beat noise effect is negligible especially in the controlled multi laser source configuration.

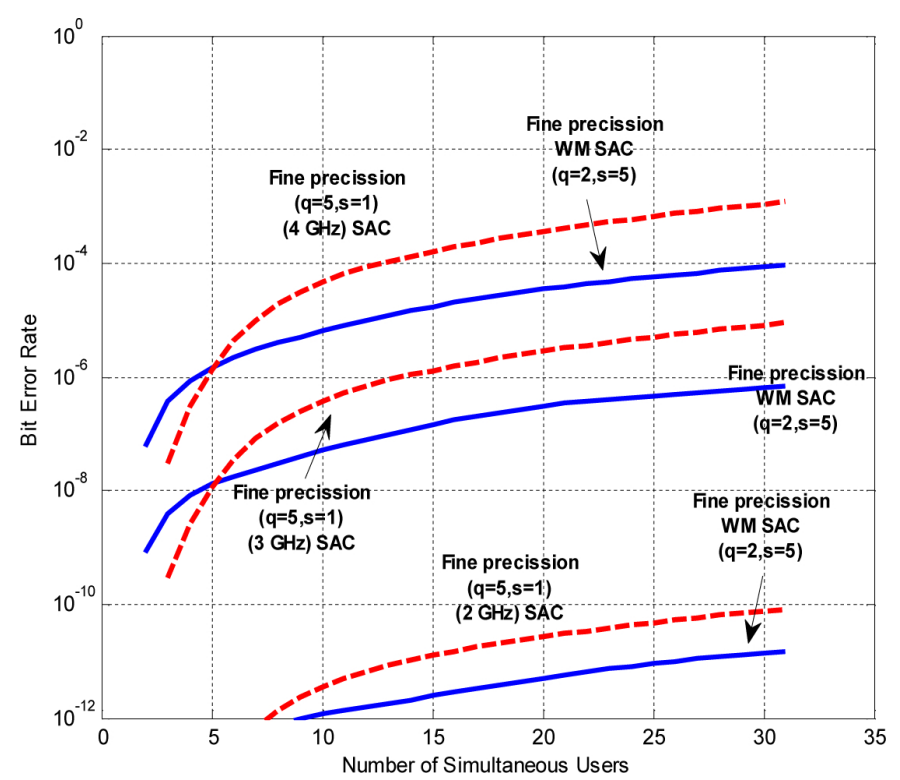

FIG. 8 BER versus the active number of users for SAC code $(q=5, s=1)$ and WM SAC code $(q=2, s=5)$ at data rate of $10 \mathrm{~Gb} / \mathrm{s}$.

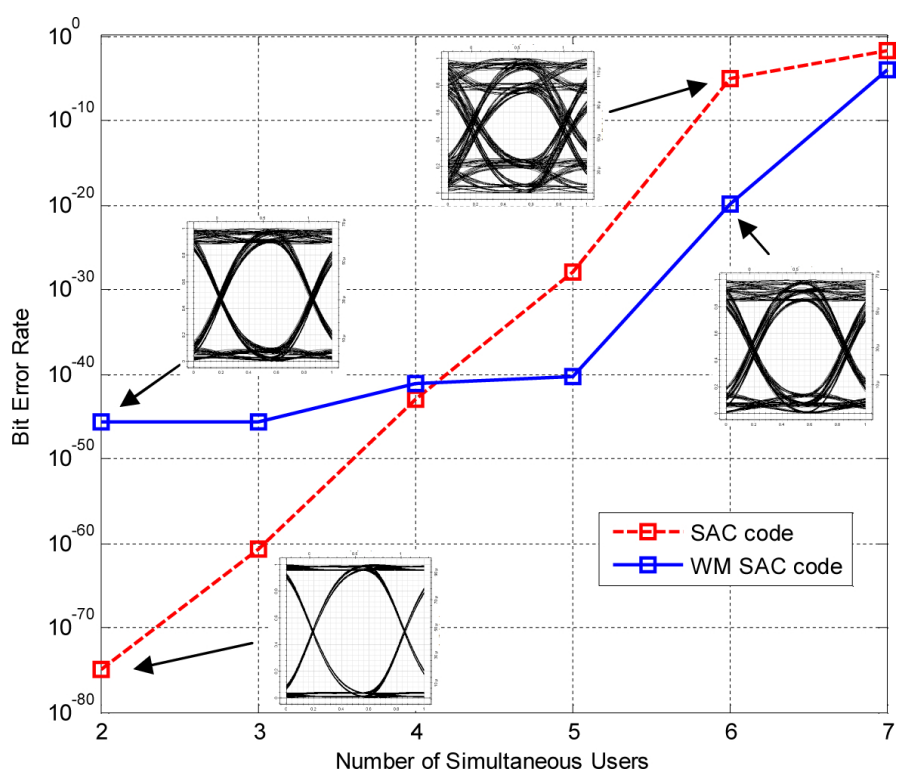

FIG. 9 BER versus the active number of users for SAC and WM SAC codes data rate $1.25 \mathrm{~Gb} / \mathrm{s}$.

The BER at different data rates for both the SAC and WM SAC codes is given in Figure 10 for six active users. Error free transmission $\left(\mathrm{BER}<10^{-9}\right)$ is assured for the WM SAC code for data rates up $2.5 \mathrm{~Gb} / \mathrm{s}$, whereas at $5 \mathrm{~Gb} / \mathrm{s}$ only $1.4 \times 10^{-6} \mathrm{BER}$ is reported. On the other hand, faulty transmission is obtained for all data rates for the SAC code, with a minimum BER of $1.1 \times 10^{-6}$ reported at $155 \mathrm{Mb} / \mathrm{s}$. It is important to note that for this system to support higher data rate (e.g. $10 \mathrm{~Gb} / \mathrm{s}$ or more), the bin spectral bandwidth must be increased. This will lead to an increase in the spacing between the adjacent lasers, which will decrease the beat noise and thus increase the data rate.

Figure 11 shows the BER versus the laser launch power for both the SAC code and the WM SAC code for six simultaneous users at $1.25 \mathrm{~Gb} / \mathrm{s}$. At low power $(<-20 \mathrm{dBm})$, thermal noise and shot noise are dominant and the BER is at its worst value for both SAC and WM SAC codes. By increas- 


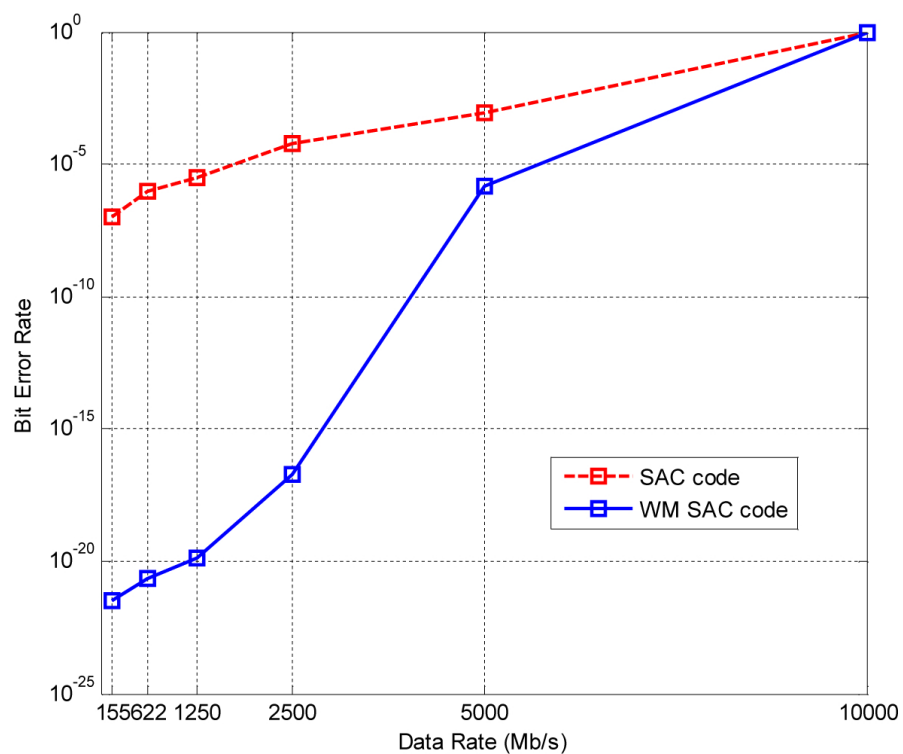

FIC. 10 BER versus the data rate for SAC and WM SAC codes for six users at data rate $1.25 \mathrm{Cb} / \mathrm{s}$.

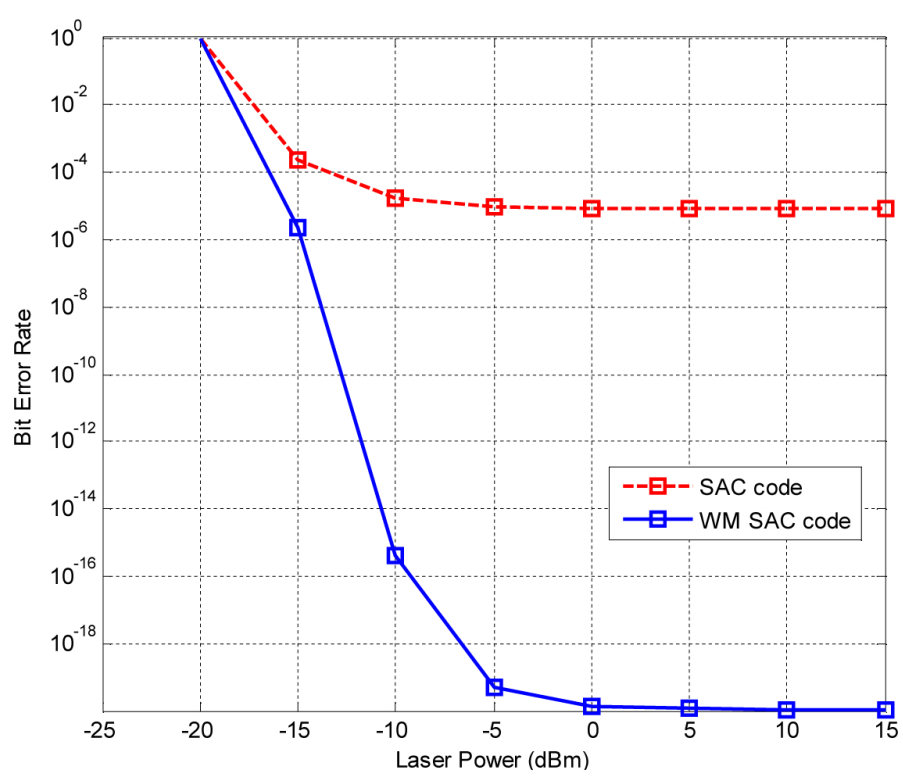

FIC. 11 BER versus laser launch power for SAC and WM SAC codes for six users at data rate $1.25 \mathrm{~Gb} / \mathrm{s}$.

ing the laser's power, the signal to noise ratio (SNR) starts to increase, leading to a better BER. With the increase in signal power, the effects of thermal and shot noise decrease and beat noise becomes the dominant source of noise. Increasing the launch power above $5 \mathrm{dBm}$ leads to a BER floor, which is due to the fact that the beat noise is a signal-dependent noise (increases with signal strength). From Figure 11 we can see for the WM SAC code a less than $-10 \mathrm{dBm}$ laser power is needed to achieve error free transmission. Meanwhile, the minimum BER reported for the SAC code is $8.15 \times 10^{-6}$ at laser launch power of at least $5 \mathrm{dBm}$.

\section{CONCLUSION}

In this paper we conducted a performance analysis on the use WM SAC codes to reduce the beat noise in laser based sources SAC OCDMA systems. The WM SAC code is a SAC code repeated diagonally in the wavelength domain. The inphase cross correlation has a maximum value of one between codes in the same group and zero with codes in different groups. Our results show that for small number of users the SAC code outperforms the WM SAC code due to the higher weight of the code. Meanwhile, as the number active users increases the WM SAC shows higher beat noise mitigation due to its better correlation properties. Results show a $57 \%$ increase in the active number users for the WM SAC over the SAC code at $1.25 \mathrm{~Gb} / \mathrm{s}$ and a two fold increase in the number of users at $10 \mathrm{~Gb} / \mathrm{s}$. Furthermore, an improvement of over ten orders of magnitude in BER is reported for six active users at $1.25 \mathrm{~Gb} / \mathrm{s}$. Apart from its improved performance, the WM SAC codes are more flexible and require less complexity in the encoder and decoder designs.

\section{References}

[1] H. Ghafouri-Shiraz, and M. Karbassian, Optical CDMA Networks: Principles, Analysis and Applications (Wiley, New York, 2012).

[2] D. Zaccarin, S. Member, and M. Kavehrad, "An Optical CDMA System Based on Spectral Encoding of LED," IEEE Photonic. Tech. L. 4, 479-482 (1993).

[3] M. Kavehrad, and D. Zaccarin, "Optical code-division-multiplexed systems based on spectral encoding of noncoherent sources," J. Lightwave Technol. 13, 534-545 (1995).

[4] Z. A. El-Sahn, B. J. Shastri, M. Z. M. Zeng, N. Kheder, D. V. Plant, and L. A. Rusch, "Experimental Demonstration of a SAC-OCDMA PON With Burst-Mode Reception: Local Versus Centralized Sources," J. Lightwave Technol. 26, 1192-1203 (2008).

[5] J. Penon, W. Mathlouthi, S. LaRochelle, and L. A. Rusch, "An Innovative Receiver for Incoherent SAC-OCDMA Enabling SOA-Based Noise Cleaning: Experimental Validation," J. Lightwave Technol. 27, 108-116 (2009).

[6] C.-C. Yang, "Spectral Efficiencies of the Optical CDMA-Based PONs Using Two-Code Keying," IEEE Commun. Lett. 14, 767-769 (2010).

[7] C. Yang, "Spectral Amplitude Coding Optical CDMA Networks Using $2^{\wedge} \mathrm{m} \times 2^{\wedge} \mathrm{m}$ Waveguide Gratings," IEEE Photonic. Tech. L. 22, 1835-1837 (2010).

[8] A. Chazisaeidi, and L. A. Rusch, "Capacity of SOA-Assisted SACOCDMA," IEEE Photonic. Tech. L. 22, 441-443 (2010).

[9] B.-C. Yeh, C.-H. Lin, and J. Wu, "Noncoherent Spectral/Spatial oCDMA System Using Two-Dimensional Hybrid Codes," J. Commun. Netw. 2, 653 (2010).

[10] M. Noshad, and K. Jamshidi, "Bounds for the BER of Codes With Fixed Cross Correlation in SAC-OCDMA Systems," J. Lightwave Technol. 29, 1944-1950 (2011).

[11] A. M. Alhassan, N. M. Saad, and N. Badruddin, "An Enhanced Detection Technique for Spectral Amplitude Coding Optical CDMA Systems," IEEE Photonic. Tech. L. 23, 875-877 (2011).

[12] H. Chen, S. Xiao, L. Yi, Z. Zhou, M. Zhu, J. Shi, et al., "A Tunable Encoder/Decoder Based on Polarization Modulation for the SACOCDMA PON," IEEE Photonic. Tech. L. 23, 748-750 (2011).

[13] H. M. H. Shalaby, "Closed-Form Expression for the Bit-Error Rate of Spectral-Amplitude-Coding Optical CDMA Systems," IEEE Photonic. Tech. L. 24, 1285-1287 (2012). 
[14] H. M. H. Shalaby, "Efficient Use of PPM in Spectral-AmplitudeCoding Optical CDMA Systems," J. Lightwave Technol. 30, 3512-3519 (2012).

[15] E. D. J. Smith, R. J. Blaikie, and D. P. Taylor, "Performance enhancement of spectral-amplitude-coding optical CDMA using pulseposition modulation," IEEE T. Commun. 46, 1176-1185 (1998).

[16] S. Ayotte, and L. A. Rusch, "A Comparison of Optical Sources for Spectral Amplitude Coding OCDMA," in Proceedings of IEEE LEOS 19th Annual Meeting, 911-912 (IEEE, Montreal, 2006).

[17] S. Ayotte, and L. A. Rusch, "Increasing the Capacity of SAC-OCDMA: Forward Error Correction or Coherent Sources?," IEEE J. Sel. Top. Quant. 13, 1422-1428 (2007).

[18] A. T. Pham, N. Miki, and H. Yashima, "Spectral-amplitude-encoding optical-code-division-multiplexing system with a heterodyne detection receiver for broadband optical multiple-access networks," J. Opt. Netw. 4, 621-631 (2005).
[19] M. Yoshino, S. Kaneko, T. Taniguchi, N. Miki, K. Kumozaki, T. Imai, et al., "Beat Noise Mitigation of Spectral Amplitude Coding OCDMA Using Heterodyne Detection," J. Lightwave Technol. 26, 962-970 (2008).

[20] Y. Yang, M. Foster, J. B. Khurgin, and A. B. Cooper, "Heterodyne detection using spectral line pairing for spectral phase encoding optical code division multiple access and dynamic dispersion compensation," Opt. Express. 20, 17600-17609 (2012).

[21] C.-C. Yang, "Hybrid Wavelength-Division-Multiplexing / SpectralAmplitude-Coding Optical CDMA System," IEEE Photonic. Tech. L. 17 1343-1345 (2005). 